\title{
Mesh implantation for pelvic organ prolapse improves quality of life
}

\author{
Atilla Yesil · Dirk Watermann $\cdot$ Juliane Farthmann
}

Received: 21 March 2013/Accepted: 4 October 2013/Published online: 17 October 2013

(C) Springer-Verlag Berlin Heidelberg 2013

\begin{abstract}
Purpose The use of alloplastic meshes for repair of female pelvic organ prolapse (POP) has recently been discussed for its indications and safety. Mesh exposure and chronic pelvic pain are among the risks that need to be addressed to the patients. The purpose of this prospective observational study is to investigate the effect of vaginal mesh implantation on pelvic floor-related quality of life (QoL).

Methods 55 patients were included in this prospective multicenter study. A validated QoL questionnaire comprising items on prolapse symptoms and bladder, bowel and sexual function was used. QoL data were acquired before and 1 year after POP surgery. Patients underwent POP surgery with implantation of either Prolift ${ }^{\circledR}$ or Seratom $^{\circledR}$ mesh.

Results Quality of life scores improved significantly after surgery. Prolapse complaints were reduced from 4.43 to $0.26(p<0.001)$, and bladder and bowel complaints improved from 3.03 to $1.46(p<0.001)$ and from 1.93 to $1.60(p<0.01)$ at follow-up. Furthermore, the sexual function score improved from 2.31 to 1.12 postoperatively $(p<0.01)$.
\end{abstract}

\footnotetext{
A. Yesil ( $\square)$

Department of Obstetrics and Gynecology, Cantonal Hospital of Baselland, Liestal, Switzerland

e-mail: atilla.yesil@ksbl.ch

D. Watermann

Department of Obstetrics and Gynecology, Diakonie Hospital, Freiburg, Germany

J. Farthmann

Department of Obstetrics and Gynecology, University Medical Center, Freiburg, Germany
}

Conclusion Despite the risks discussed for vaginal mesh repair, we observed a statistically significant improvement of pelvic floor-related quality of life of POP patients.

Keywords Pelvic organ prolapse - Polypropylene mesh · Quality of life · Sexuality

\section{Introduction}

The incidence of pelvic organ prolapse (POP) among women is high [1]. Up to $60 \%$ of women suffer from different forms of genital prolapse, mainly due to pregnancy and vaginal delivery [2]. Another predisposing factor is continuous intra-abdominal pressure, for example due to chronic bronchitis or obesity [3-6]. The resulting complaints are versatile and often accompanied by urinary incontinence representing a huge burden for women. Urinary tract dysfunction, urge symptoms and recurrent urinary tract infection can arise as a result of POP [7]. The implications on patients' quality of life and sexuality can be far-reaching. Women may refrain from sexual activity due to prolapse symptoms. However, many women do not seek help from their physicians as they feel ashamed [8].

Previous studies showed recurrence rates of up to $40 \%$ after native tissue repair of POP [9-11]. POP surgery has experienced a fundamental change by the establishment of vaginal meshes. Meshes have been widely used; however, few studies are currently available regarding long-term results. Some studies showed improved QoL scores after mesh implantation [10]. An often raised criticism about vaginal mesh implantation is the risk of dyspareunia and chronic pelvic pain. Another topic of discussion is whether SUI treatment should be conducted simultaneously or after POP surgery. The prevalence of de novo SUI after POP 
surgery was reported between 0 and $12 \%$ in different studies [12-15].

Most of the previous studies defined success of POP surgery as postoperative reconstruction of the defect and improvements in general symptoms. Subjective recovery and sexuality were investigated as the secondary subjects in these studies. In our study, the patients' pelvic floorrelated quality of life was assessed pre- and postoperatively.

The overall aim of this prospective, multicenter study was to evaluate the implications of vaginal mesh implantation on patients' quality of life and sexuality 1 year after the operation.

\section{Materials and methods}

The study was conducted between 2007 and 2009. 60 patients undergoing surgery for POP either at the Department of Obstetrics and Gynecology at the University Medical Center Freiburg or the Department of Obstetrics and Gynecology at the Hospital of Waldshut in Germany were prospectively recruited for the study. All patients had symptomatic anterior and/or apical prolapse $\geq 2$ (see Table 1). The indication for the use of mesh was based on the surgeon's decision, either due to previous surgery, considerable lateral defect or risk factors for recurrent prolapse (obesity, chronic obstructive pulmonary disease, etc.). The choice of the type of mesh used was made by the surgeon. The meshes were those commonly used at the participating hospitals. The ethical approvals were obtained from ethical committees of the University of Freiburg and Medical Association for the State of Baden-Württemberg.

\section{Surgery}

Preoperative estrogen treatment was administered to postmenopausal patients. Single-shot antibiotic therapy was given. Both implants used (Prolift ${ }^{\circledR}$, Gynecare Johnson \& Johnson, Baltimore, USA and Seratom ${ }^{\circledR}$, Serag Wiessner KG, Naila, Germany) are made from macroporous, monofilament polypropylene. The anterior meshes had four arms for the transobturator approach; in case of the total mesh

Table 1 Preoperative prolapse compartment $(n=55)$

\begin{tabular}{|c|c|c|c|c|c|c|}
\hline \multirow[t]{2}{*}{ Prolapse stage } & \multicolumn{2}{|c|}{ Anterior } & \multicolumn{2}{|c|}{ Combined } & \multicolumn{2}{|c|}{ Posterior } \\
\hline & $n$ & $\%$ & $n$ & $\%$ & $n$ & $\%$ \\
\hline Stage I & 1 & 1.8 & 1 & 1.8 & 0 & 0 \\
\hline Stage II & 10 & 18 & 1 & 1.8 & 0 & 0 \\
\hline Stage III & 25 & 45.5 & 13 & 23.6 & 1 & 1.8 \\
\hline Stage IV & 0 & 0 & 3 & 5.5 & 0 & 0 \\
\hline
\end{tabular}

(Prolift ${ }^{\circledR}$ ) and of the posterior mesh, fixation was through the sacrospinous ligament. After colpotomy and dissection of the endopelvic fascia, the meshes were implanted using the devices provided by the manufacturers. Excessive tissue of the vaginal wall was not resected to prevent mesh exposure. After implantation of the mesh, the vaginal wall was closed using a running suture.

Quality of life questionnaire and evaluation of sexual function

All patients scheduled for mesh implantation in one of the two hospitals were asked to participate in the study. Informed consent was obtained from all women. QoL data were acquired before and 1 year after surgery. Patients filled out the "German pelvic floor questionnaire" [16], covering items on prolapse symptoms and bladder, bowel and sexual function.

Statistical analysis

The statistical software SPSS ${ }^{\circledR}$ Version 17.0 was used to analyse the data. Frequency analysis was computed to get descriptive information. Median, standard deviation, minimum and maximum values were calculated for each case. The pre- and postoperative scores of each function were compared. In addition pre- and postoperative total scores were calculated and compared. Paired $t$ test was used to test the difference between preoperative and postoperative scores.

\section{Results}

Localization of prolapse and surgery

Table 1 depicts the distribution of the POP stages in the study population. Apart from mesh implantation, the following additional procedures were performed: anterior colporrhaphy (36 patients), combined anterior and posterior colporrhaphy (18 patients) and one posterior colporrhaphy with mesh insertion. Table 2 displays the types of mesh used.

Table 2 Type of mesh used

\begin{tabular}{|c|c|c|c|c|c|c|}
\hline & \multicolumn{2}{|c|}{ Anterior } & \multicolumn{2}{|c|}{ Combined } & \multicolumn{2}{|c|}{ Posterior } \\
\hline & $n$ & $\%$ & $n$ & $\%$ & $n$ & $\%$ \\
\hline Gynecare Gynemesh PS Prolift ${ }^{\circledR}$ & 9 & 16 & 10 & 18 & 1 & 1.8 \\
\hline Seratom $^{\circledR}$ & 27 & 49 & 8 & 14 & 0 & 0 \\
\hline
\end{tabular}


Patients' demographics

Of the 60 patients included in the study, 55 could be taken for analysis. Median follow-up time was 12.4 months. The response rate was $91 \%$ : two patients were excluded from the study as they filled out the questionnaire only after surgery. Three more did not fill out the questionnaire after 1 year. The median age was 64.3 years $(\min =44$, $\max =81$ years $)$. The mean number of vaginal deliveries was 2.46 . Of the patients $2(3 \%)$ were nulliparous, $7(13 \%)$ had one vaginal birth, 44 (80 \%) 2-4 vaginal births and $2(4 \%)$ experienced more than five births.

Sexual activity

Of the 55 patients, $13(24 \%)$ were regularly and 17 (31\%) seldom sexually active before the operation. 25 women $(45 \%)$ were not sexually active preoperatively. The reasons for being not sexually active were as follows: $8(31 \%)$ patients did not have a partner, $7(27 \%)$ had an impotent partner, $2(8 \%)$ were not interested in sexual intercourse, $1(4 \%)$ had impaired lubrication, 6 $(11 \%)$ were embarrassed due to incontinence or prolapse, 1 (4\%) felt 'very' old, 1 (4\%) due to other reasons. Sexual activity rates and the reasons for inactivity before and after the treatment are depicted in Tables 3 and 4.

Table 3 Sexual activity

\begin{tabular}{llllll}
\hline & \multicolumn{2}{l}{ Preoperative } & & \multicolumn{2}{l}{ Postoperative } \\
\cline { 2 - 3 } & $n$ & & & $n$ & $\%$ \\
\hline Not active & 25 & 45.5 & & 25 & 45.5 \\
Seldom & 17 & 30.9 & & 17 & 30.9 \\
Regularly & 13 & 23.6 & & 11 & 20.0 \\
\hline
\end{tabular}

Table 4 The reason of being not sexually active

\begin{tabular}{lll}
\hline & $\begin{array}{l}\text { Preoperative } \\
(n=25)\end{array}$ & $\begin{array}{l}\text { Postoperative } \\
(n=25)\end{array}$ \\
\hline No partner & 8 & 8 \\
Partner unable & 7 & 7 \\
Not interesting in sexuality & 2 & 4 \\
Vaginal dryness & 1 & - \\
Embarrassment due to prolapse/ & 6 & - \\
$\quad$ incontinence & & 1 \\
Pain & - & - \\
Other & 1 & 5 \\
Not specified & - & \\
\hline
\end{tabular}

Dyspareunia

Frequency of dyspareunia was also explored by the statements of patients on a four point scale (never, seldom, mostly and always). Localization of dyspareunia $(n=12)$ preoperatively was explored with further questions. Five patients $(42 \%)$ felt pain during sexual intercourse at the introitus of the vagina, three patients $(25 \%)$ deep inside the pelvis, three patients $(25 \%)$ at the introitus and deep inside, and one patient $(8 \%)$ made no statement about the localization.

Localization of dyspareunia $(n=10)$ postoperatively was stated as follows: two (20\%) felt pain during sexual intercourse at the introitus, five patients (50\%) deep inside the pelvis, two patients $(20 \%)$ at the introitus of the vagina and deep inside, and one patient $(10 \%)$ made no statement about the localization. Three $(11 \%)$ patients reported de novo dyspareunia. The difference of the pre- and postoperative values did not show statistical significance.

Incontinence and bladder function

54 patients could be evaluated regarding urinary incontinence. The effect of incontinence on QoL was explored by a four point scale. 36 patients $(67 \%)$ complained about urge incontinence before surgery. While 22 patients (41\%) still expressed suffering, $14(26 \%)$ stated improvement 1 year after the operation, however, this was not statistically significant. 30 patients (54\%) expressed complaints about stress urinary incontinence (SUI) before the operation, which was improved in $19(35 \%)$ postoperatively (n.s.). Four (7\%) patients showed de novo urge incontinence and $7(12 \%)$ de novo SUI.

Bladder function complaints were explored by different statements about 'slow stream of urine', 'not completely emptied bladder' or need to 'press to urinate'. Apart from the improvement of incontinence problems, bladder function was also positively changed after 1 year: the differences between pre- and postoperative statements, 'incomplete emptying of bladder' $(p<0.001)$; 'press to urinate' $(p<0.05)$ and 'slow stream of urine' $(p<0.001)$, were statistically significant. For details see Tables 5 and 6 .

Table 5 Bladder function

\begin{tabular}{lrrrrrrl}
\hline & \multicolumn{2}{c}{ Preoperative } & & \multicolumn{2}{l}{ Postoperative } & $p$ \\
\cline { 2 - 3 } & $n_{1}$ & $n_{2}$ & & $n_{1}$ & $n_{2}$ & \\
\hline $\begin{array}{l}\text { Incomplete emptying of } \\
\quad \text { bladder }\end{array}$ & 14 & 38 & 34 & 21 & $<0.001$ \\
$\begin{array}{l}\text { Need to press to urinate } \\
\text { Slow stream of urine }\end{array}$ & 32 & 23 & & 46 & 8 & $<0.05$ \\
& 9 & 44 & & 27 & 24 & $<0.001$
\end{tabular}

$n_{1}$ 'never'; $n_{2}$ 'sometimes', 'often', 'always' 
Table 6 Psychological strainbladder function complaints

$n_{1}$ "not at all"; $n_{2}$ "slightly",

"moderately"; $n_{3}$ "extremely"

\begin{tabular}{|c|c|c|c|c|c|c|}
\hline & \multicolumn{2}{|l|}{$n_{1}$} & \multicolumn{2}{|l|}{$n_{2}$} & \multicolumn{2}{|l|}{$n_{3}$} \\
\hline & Preoperative & Postoperative & Preoperative & Postoperative & Preoperative & Postoperative \\
\hline $\begin{array}{l}\text { How do } \\
\text { bladder } \\
\text { complaints } \\
\text { affect you? }\end{array}$ & 9 & 16 & 10 & 18 & 1 & 1.8 \\
\hline
\end{tabular}

Table 7 Quality of life score pre- and postoperatively $(n=55)$

\begin{tabular}{llll}
\hline & Preoperative & Postoperative & $p$ \\
\hline Prolapse score & 4.43 & 0.26 & $<0.001$ \\
Bladder function score & 3.03 & 1.46 & $<0.001$ \\
Bowel function score & 1.93 & 1.60 & $<0.01$ \\
Sexual function score & 2.31 & 1.12 & $<0.01$ \\
Total score & 0.26 & 0.05 & $<0.001$ \\
\hline
\end{tabular}

Quality of life scores

In all four aspects of the questionnaire we could observe statistically significant improvement of quality of life scores 1 year after surgery. The mean preoperative bladder function score improved from 3.03 to 1.46 after the surgery $(p<0.001)$, prolapse score $4.43(\mathrm{SD}=1.96)$ to 0.26 $(\mathrm{SD}=0.62)$ postoperatively. Bowel function score changed from 1.93 to 1.60 postoperatively $(p<0.01)$. Furthermore, also sexual function score showed an improvement from 2.31 to $1.12(p<0.01)$. Table 7 shows the pre- and postoperative QoL scores.

\section{Discussion}

We present a prospective multicenter study on the effects of vaginal mesh implantation on patients' quality of life and sexuality. Special attention was paid to the changes in bladder function after the operation. Some of the findings regarding the comparison between pre- and postoperative values are not statistically significant, however, a positive trend could be observed.

The relationship between POP and sexual function is discussed controversially, some studies describe a close relationship [17], whereas others do not see this correlation [18, 19]. Patients undergoing POP surgery are often postmenopausal, but this does not mean that sexual function can be left out of sight when counseling women on POP repair [20].

Apart from problems related to POP itself, pelvic floor repair, no matter whether with native tissue or alloplastic mesh, may result in dyspareunia [21]. About half of our study population was not sexually active, in most cases due to partner problems (no partner/partner sick or impotent). Pain was only seldom named as reason for inactivity. In our population, we observed a statistically significant improvement of sexual function score; however, the percentage of patients sexually active before and after the operation was almost similar. Nearly a quarter of the patients stated feeling ashamed due to POP as the reason for not being sexually active. Of those, $12 \%$ were sexually active 1 year after the operation, which may be due to an improved body image. This finding shows that the improvement is in the qualitative aspect rather than the numbers of the sexually active women.

Furthermore, an improvement of dyspareunia was observed in $17.8 \%$ of our patients, with a rate of de novo dyspareunia of $10.7 \%$. To better evaluate pelvic floor pain and not only dyspareunia, a visual analog scale could be useful for further studies.

25.9 and $20 \%$ of the patients stated improvements of urge or stress incontinence complaints. This of course is only a subjective assessment, as it was not confirmed by urodynamic testing. Improvement of incontinence complaints can be explained by stress-free suspension of the urethra by mesh implantation. The $12 \%$ rate of de novo SUI is in accordance with other recent studies $[12,22]$. This again strengthens that a mid-urethral sling should rather be implanted in a second operation to avoid unnecessary treatment. Otherwise, too many patients would receive a sling without needing it. Also the rate of de novo urge incontinence is in accordance with other studies.

In our study, pelvic floor-related QoL scores were significantly improved 1 year after the operation. Similar improvements in the QoL scores were observed in other studies [10].

Summing up, we could observe positive effects of vaginal mesh implantation on patients' quality of life 1 year after surgery. Sexual function of our patients was not impaired after surgery. These results point out that, apart from better anatomical results of vaginal mesh implantation compared to native tissue repair the subjective outcome is satisfying. However, it should be tested in randomized studies whether there is a difference in QoL outcome between the two techniques. As vaginal mesh implantation might have serious complications, the operation should not be performed uncritically. It should only be carried out after thoroughly counseling the patients and explaining possible alternatives. 
Conflict of interest None.

\section{References}

1. Mallett VT, Bump RC (1994) The epidemiology of female pelvic floor dysfunction. Curr Opin Obstet Gynecol 6(4):308

2. Dannecker C, Anthuber C (2000) The effects of childbirth on the pelvic-floor. J Perinat Med 28(3):175

3. Fritel $X$ et al (2009) Symptomatic pelvic organ prolapse at midlife, quality of life, and risk factors. Obstet Gynecol 113(3):609

4. Whitcomb EL et al (2009) Prevalence and degree of bother from pelvic floor disorders in obese women. Int Urogynecol J 20(3):289-294

5. Swift S et al (2005) Pelvic Organ Support Study (POSST): the distribution, clinical definition, and epidemiologic condition of pelvic organ support defects. Am J Obstet Gynecol 192(3): 795-806

6. Kudish BI et al (2009) Effect of weight change on natural history of pelvic organ prolapse. Obstet Gynecol 113(1):81

7. Jelovsek JE, Maher C, Barber MD (2007) Pelvic organ prolapse. Lancet 369(9566):1027-1038

8. Athanasiou S et al (2012) Pelvic organ prolapse contributes to sexual dysfunction: a cross-sectional study. Acta Obstet Gynecol Scand 91(6):704-709

9. Altman D et al (2011) Anterior colporrhaphy versus transvaginal mesh for pelvic-organ prolapse. $N$ Engl J Med 364(19): $1826-1836$

10. Nieminen K et al (2008) Symptom resolution and sexual function after anterior vaginal wall repair with or without polypropylene mesh. Int Urogynecol J 19(12):1611-1616

11. Maher C, Baessler K (2006) Surgical management of anterior vaginal wall prolapse: an evidence based literature review. Int Urogynecol J 17(2):195-201
12. Abdel-fattah M, Ramsay I (2008) Retrospective multicentre study of the new minimally invasive mesh repair devices for pelvic organ prolapse. BJOG 115(1):22-30

13. Fatton B et al (2007) Transvaginal repair of genital prolapse: preliminary results of a new tension-free vaginal mesh (Prolift ${ }^{\mathrm{TM}}$ technique) - a case series multicentric study. Int Urogynecol J 18(7):743-752

14. Solà Dalenz V et al (2007) Treatment of female genital prolapse with Prolift system. Acta Urol Esp 31(8):850-857

15. Cosson $\mathrm{M}$ et al (2005) Prolift mesh (Gynecare) for pelvic organ prolapse surgical treatment using the TVM group technique: a retrospective study of 687 patients. Neurourol Urodyn 24(5/ 6): 121

16. Baessler K (2006) Psychometrische Validierung eines umfassenden Beckenboden-fragebogens für Klinik, Praxis und Forschung. Geburtshilfe und Frauenheilkunde 67(S1):01-10

17. Butler R et al (1994) Love and sex after 60: how to evaluate and treat the sexually-active woman. Geriatrics 49(11):33

18. Weber AM et al (1995) Sexual function in women with uterovaginal prolapse and urinary incontinence. Obstet Gynecol 85(4):483-487

19. Fashokun TBO et al (2013) Sexual activity and function in women with and without pelvic floor disorders. Int Urogynecol J 24(1):91-97

20. Gass MLS et al (2011) Patterns and predictors of sexual activity among women in the hormone therapy trials of the Women's Health Initiative. Menopause 18(11):1160

21. Dua A et al (2012) The effect of prolapse repair on sexual function in women. J Sex Med 9:1459-1465

22. Aungst MJ et al (2009) De novo stress incontinence and pelvic muscle symptoms after transvaginal mesh repair. Am J Obstet Gynecol 201(1):73 e1-7 